\title{
SELIG- UND HEILIGSPRECHUNG VON PATER M. M. KOLBE UND DER CHRISTLICHE MÄRTYRERBEGRIFF*
}

Am 2. November 1982, während der Unterzeichnung des Vertrags über die wissenschaftliche Zusammenarbeit zwischen der Abteilung für katholische Theologie der Ruhr-Universität Bochum und der Päpstlichen Theologischen Hochschule Kraków, sagte der Rektor Prof. Dr. K. Ipsen u. a., daß der Vertrag der Wiederherstellung der guten Beziehungen zwischen dem polnischen und dem deutschen Volk angesichts der grauenhaften Vergangenheit dienen sollte. Es besteht kein Zweifel darüber, daß bei der Wiederherstellung dieser Beziehungen die Gestalt des hl. Maximilians Kolbe eine besondere Rolle spielt. Und selbstverständlich habe ich diese Tatsache bei der Themawahl für den folgenden Vortrag berücksichtigt.

Dennoch erfordert dieses Thema hinsichtlich des Ortes und des Publikums vor dem es dargestellt wird (Ruhr-Universität Bochum: 22 XI 1983), eine besondere Behandlung. Auf jeden Fall kommt hier keinerlei Hagiographie in Frage. Auf der Grundlage des Beatifikations- und Kanonisationsverfahrens des $P$. Kolbe, werde ich versuchen, die theologisch-moralische Problematik des christlichen Martyriums darzustellen, die damals zum Vorschein kam. Dieses Problem ist zugleich ein gutes Beispiel für die Entwicklungsdynamik der katholischen Theologie und der Lehre des Magisteriums.

Während der Feierlichkeiten der Seligsprechung am 17.10.1971 wurde „,auf Grund apostolischer Gewalt und Autorität der Ehrwürdige Diener Gottes Maximilian Maria Kolbe aus dem Orden der Minder Brüder in die Reihe der Seligen eingetragen". In der Seligsprechungsformel des Apostolischen Stuhles wurde also dem Seligen Kolbe der Titel eines Märtyrers nicht zuerkannt ${ }^{1}$. Ein diskretes Schweigen wahrt zu diesem Titel auch das Messformular. Die Oration, die Sekret und die Postkommunion der ersten, vom Papst Paul VI. in der Basilika des hl. Petrus

* O przyznanie O. M. M. Kolbemu tytułu męczennika upominał się autor na terenie RFN już 4 i 6 listopada 1981 r. w wykładach, jakie wyglosil wówczas na uniwersytetach $w$ Moguncji i Ratysbonie. Wykład miniejszy nawiązuje do nich. Por. jeszcze wcześniejszy tekst autora na ten temat: Wyznawca czy męczennik, „Tygodnik Powszechny", 9 VII 1972, s. 7 i w Miles Immaculatae, 10 (1974) 288-295.

1 „L'Osservatore Romano”, 18-19 Ottobre 1971, S. 3. 
zur Ehrung P. Kolbes gelesenen Messe, nennen nur den ,seligen Maximilian Maria Kolbe".

Im Gegensatz dazu sagte am 10.10.1982 in der Heiligsprechungsformel Papst Johannes Paul II. auf dem St. Petrus-Platz: „Nachdem wir viele Brüder im Bischofsamt nach dem Rat gefragt hätten, (...) tragen wir den Pater Maximilian Maria Kolbe in den Heiligenkatalog ein, und beschließen ihn in der ganzen Kirche pflichtmässig als heiligen Märtyrer zu verehren". ${ }^{2}$ Die Oration der hl. Messe, die damals vom Papst gelesen wurde, nennt den P. Kolbe in Einklang mit dieser Entscheidung, „Priester und Märtyrer".

Es unterliegt keinem Zweifel, daß wir hier mit einem besondern Fall zu tun haben. In bin außerstande $\mathrm{zu}$ beurteilen, ob in der Seligund Heiligsprechungspragmatik der römisch-katholischen Kirche ähnliche Fälle jemals vorgekommen sind. ES geht hier um eine Änderung oder eher, Erweiterung der Entscheidung des. Lehramtes hinsichtlich der Zuerkennung dem Pater Kolbe des ältesten und zugleich würdigsten Titels eines Märtyrers. Das Zögern des Apostolischen Stuhles in dieser Frage kann wohl auf verschiedene Faktoren zurückgeführt werden, unter denen sicherlich eine der entscheidenden Ursachen die unterschiedliche Einstellung und verschiedene Auffassung des christlichen Martyriums selbst ist. Daher wollen wir wenigstens ganz knapp sagen, was das christliche Martyrium in seinem Wesen ist, bevor wir zur Darstellung des Märtyrertums des Paters Kolbe übergehen.

1. Es scheint, daß Origenes in seinem Kommentar zum Johannesevangelium gut den Sinn des christlichen Martyriums aufgefasst hat. Seiner Meinung nach kann jeder, der der Wahrheit Zeugnis gibt, abgesehen davon ob er das durch Worte, Taten oder in irgendwelcher Weise tut mit Recht ein Zeuge genannt werden. Es ist zu einem Brauch bei den Brüdern geworden, dank der Bewunderung für diejenigen, die bis zum Tod für Wahrheit oder Unschuld gekämpft haben - als Zeugen (Märtyrer) im vollen Sinn ausschliesslich diese Menschen zu nennen, die vergiessend ihr Blut ein Zeugnis dem Geheimnis des Glaubens gegeben haben. Der Heiland sieht jedoch jeden, der vom seiner Lehre Zeugnis gibt, als Zeugen an ${ }^{3}$.

Die Meinungen über das Martyrium der zeitgenössischen Autoren decken sich ganz mit diesen Worten. Zum Beispiel R. Reitzenstein schreibt über das Martyrium des hl. Polykarp und über das in der ganzen Patristik dargestellte Martyrium so: den Menschen, der für Christus gestorben ist, hat man martys tou Christou oder martys tou Theou genannt, der Tod wurde als Martyria oder Martyrion angesehen, gestor-

z "L'Osservatore Romano", 11-12 Ottobre 1982, S. 4.

3 Orig èn e, Commentaire sur saint Jean, II, 210: Sources Chr. 120, 351. 
ben sein heisst martyresai ". Ähnliche Ansicht vertritt H. Leclerq. Indem er acht Fragmente aus der Beschreibung des Martyriums des hl. Polykarp anführt, behauptet er, daß man den Sinn des Wortes Märtyrer als Bezeichnung des für den Glauben erittenen Todes nicht mehr in Zweifel ziehen darf. ${ }^{5}$ In diesem Sinn ist der Terminus Martyrium in den Wortschatz der Christen eingegangen. Ihn verwenden auch Schriftsteller, deren Werke nur den Lokalkirchen bekannt sind.

Hieraus ergibt sich, daß zwei Faktoren das Wesen des Martyriums in christlicher Auffassung bilden, und zwar: das Christo öffentlich gegebene Zeugnis und der bewusst und freiwillig angenommene Tod, um es zu bestätigen. Dabei sind sie in gleicher Weise notwendig, d.h. ohne sie gibt es einfach keine christliche Martyriumsidee.

Dessenungeachtet darf man die grundsätzliche Rolle des Inhaltes des von den Märtyrern gegebenen Zeugnisses nicht vergessen. Bekannt sind nämlich die Worte des hl. Augustinus zu diesem Problem: „Die Ursache und nicht die blosse Strafe macht den Menschen zum Märtyrer" (Martyres non facit poena, sed causa. Nam si poena martyres faceret, omnia metalla martyribus plena essent, omnes catenae martyres traherent, omnes, qui gladio feriuntur, coronarentur). ${ }^{6}$ Mit anderen Worten sollen die christlichen Märtyrer Zeugen für Jesus von Nazareth, den Gekreuzigten und Auferstandenen sein, der lebt und "kommen wird zu richten die Lebenden und Toten". Folglich zeugen sie von der göttlichen Offenbarung im Worte, das Fleisch geworden ist (cf. J 1, 14). Also nicht nur der Jesus der Geschichte, aber Jesus Christus, der Herr, der jetzt lebt und zur Rechten des Vaters sitzt, der „Gottes Sohn kundgetan mit Gottes Allmacht" (Röm 1,4), ist Gegenstand des von den Märtyrern abgelegten Zeugnisses.

Außerdem ist hier an das Urteil des hl. Thomas von Aquin zu erinnern, das in der Theologie allgemeine Annahme gefunden hat: man leidet für Christus sowohl, wenn dies wegen des Glaubens an ihn geschieht, als auch dann, wenn man wegen guter, aus Liebe zu Christus getaner Werke verfoolgt wird. Das Christo gegebene Zeugnis umfasst also sowohl Akte des Glaubens, als auch der Sittlichkeit. Einerseits kann daher niemand ein christlicher Märtyrer genannt werden, der einer beliebigen Wahrheit Zeuge steht, sondern nur der Zeuge der göttlichen Wahrheit. Andererseits aber kann nicht nur allein der Glaube Ursache des Martyriums sein, sondern auch jede Tugend, insofern sie in einer Beziehung

- R. Reitzenstein, Bemerkungen zur Martyrienliteratur. I. Die Bezeichnung Märtyrer, in: Nachrichten von der königlichen Gesellschaft der Wissenschaften zu Göttingen. Philologisch-historische Klasse, 3 (1916) 421. Vgl. ibidem SS. 418-419, 442-445.

5 H. L e clerq, Saint, DALC, 15 (1950) 389.

6. A u g u stinus, Enarrationes in Ps. 34: PL, 36, 340. 
zu Gott steht, da sie dadurch eine gewisse Art Glaubensbekenntnis bildet. Denn der Glaube lehrt, was Gott von den Menschen verlangt und wie er sie belohnt?

Die Eigenart der christlichen Auffassung des Martyriums beruht auf ihrem christozentrischen Charakter, der in einer tiefen und personalen Verbindung zwischen Christus und dem Märtyrer besteht. Diese besondere Verbindung kann in zwei Worten zusammengefasst werden: „Nachahmung und Vereinigung".

Das ganze Christentum war immer von dem Gedanken durchgedrungen, daß das Martyrium die vollkommene Nachahmung Christi bildet. Jesus Christus ,der getreue Zeuge”, ,der treue und wahrhaftige Zeuge” (Geh. Offenb. 1, 5, cf. 3, 14) ist das Urbild des Märtyrers. In der Auffassung der Gläubigen tritt der Märtyrer durch die erlittenen Qualen an die Seite Christi des ersten Märtyrers, und eifert ihm nach. Indem er mit seinem Meister gemainsam leidet, bildet er in sich seine Marter ab und trägt ihre Merkmale. Dies ist die tiefste Verwirklichung der existentiellen Gemeinschaft mit Christus und der Höhepunkt der Liebe. Das Sterben des Christen zum Zeugnis für Jesus Christus ist demnach der Gipfel der Nachfolge Christi - protomartyris.

Von der Nachahmung zur Vereinigung mit Christus führt nur noch ein kleiner, weiterer Schritt. Das Martyrium wurde immer als der sicherste Weg zur Vereinigung mit Christus betrachtet. Es findet hier eine Vereinigung des Märtyrers mit Christus in einem besonderen Sinne statt. Auf Erden überragt sie jede andere für die Gläubigen erreichbare Vereinigung. Sie ist so innig, daß sie dem Märtyrer eine besondere Gegenwart Jesu bringt und zum Ertragen der Leiden des Martyriums Kraft verleiht. Es ist einfach so, daß Christus gemeinsam mit dem Märtyrer und in ihm lebt und wirkt. Diese Einheit des Märtyrers mit Christus wird auch nicht von dem Tod gesprengt. Der Himmel, der für viele, und nach der Meinung zahlreicher Autoren der christlichen Antike für alle, auf lange Zeit unzugänglich bleibt, öffnet sich sogleich nur für Märtyrer, ohne sie warten zu lassen. Im Augenblick des Todes erreichen sie sogleich den Herrn ${ }^{8}$.

Es wirft sich noch die Frage auf, inwiefern das II. Vaticanum diese Theologie des Martyriums ergänzt hat. Denn das Konzil berührte gelegentlich auch dieses Thema. Bestimmt betonen die Konzilstexte die Wahrheit, daß das Martyrium etwas mehr ist, als eine nur mit menschlicher Kraft vollbrachte Tat. Es ist eine ausserordentliche Gabe und die höchste Prüfung der Liebe, die nicht ausschliesslich aus eigener Initia-

7 S. T hom as, Sum. theol., II-II, q. 124, a. 5, ad 2. $78-85$.

$8 \mathrm{~A}$. Kubiś, La théologie du martyre au vinglième siècle, Roma 1968, SS. 
tive unternommen werden kann. Bei aller Bewertung des persönlichen Einsatzes des Menschen ist im Martyrium doch alles eine Gnade Gottes. Der Mensch wird zum Märtyrer, nicht dank eigener Uberzeugung und eigenem Verlangen, sondern dadurch, daß Christus ihn mit dieser groBen Würde bedacht hat ${ }^{\text {. }}$.

Gläubige Christen müßen - nicht alle in demselben Grade - zur Annahme des Martyriums bereit sein, denn unter gewissen Umständen kann es die einzige, von Gott geforderte Alternative sein. Manchmal ist es auch so, da $\beta$ das Martyrium, ohne eine sittliche Forderung unter der Androhung der ewigen Verdammnis zu sein, doch von einem Menschen aus anderen Motiven spontan unternommen wird, z. B. in der Missionsarbeit oder aus Nächstenliebe.

2. Der hier skizzierte Begriff des Martyriums, obwohl er auf den ersten Blick klar und einfach erscheint, wirft bei näherem Ansehen manche Schwierigkeiten auf. Es ist immer leichter etwas allgemein zu definieren, als in Anlehnung an eine solche Definition seine aktuelle Existenz festzustellen.

Die formale Ursache des Martyriums ist natürlich das wichtigste, da sie die christliche Hingabe begrifflich bestimmt, von anderen heroischen Akten dieser Art unterscheidet, und den übernatürlichen Charakter und Verdienstwert hervorhebt. Anders gesagt, muß das Martyrium ,organisch" mit der christlichen Offenbarung verbunden sein, das bedeutet jedoch nicht, daß beiderseits, d.h. beim Verfolgten sowohl wie beim Verfolger nicht auch andere zusätzliche Motive und Ziele mitwirken können. Es genügt, daß sie die wesentliche christliche Bedeutung der Hingabe nicht ausschliessen. Es ist auch nicht wichtig, wer der Tötende ist. Es kann sogar sein, daß er ansonsten ein guter Christ wäre. Im Falle des Martyriums kommt nur in Betracht der Hass zum Menschen wegen seiner inneren Einstellung als eines treuen Nachfolgers Christi, besonders in seinem Leiden und Sterben. Ein äusseres Zeichen beruht darin, daß der Tod nicht nur wissentlich und freiwillig, sondern auch mit Würde, Ruhe und Demut, aus übernatürlichen Gründen, angenommen wird.

Was den Pater Maximilian Kolbe anbetrifft, so ist schon vom Papst Paul VI. in seiner Beatifikationshomilie eindeutig festgestellt worden, daß Maximilian Kolbe seine Erhebung auf die Altäre hauptsächlich (principalmente) dem hehren Epilog seines Lebens, d.h. dem heroischen Tod im KZ Auschwitz verdankt. ${ }^{10}$

In einer gekürzten Beschreibung, die aufgrund der Aussagen von mehr als zehn Augenzeugen, die in den Jahren 1945-1967 gemacht

" „Lumen gentium”, $\mathrm{Nr} 15,42$. - Vgl. „Unitas redintegratio”, $\mathrm{Nr} 4$.

10 "L'Osservatore Romano", 18-19 Ottobre 1971, 1. 
wurden, hatte dieser heroische, durch die Flucht einiger Lagerhäftlinge verursachte Tod, folgenden Verlauf: 1) Die Lagerleitung wählt zehn Häftlinge, die getötet werden sollen, unter ihnen den Franz Gajowniczek; 2) Gajowniczek äussert laut seine Verzweiflung; 3) P. Kolbe tritt aus der Reihe der Häftlinge heraus; 4) Er spricht mit dem Lagerführer Fritsche; 5) Er zeigt mit der Hand auf Gajowniczek; 6) Gajowniczek wird befreit und kehrt in die Reihe der Häftlinge zurück; 7) P. Kolbe wird den zum Tode bestimmten zugereiht; 8) Die zum Tode bestimmten werden in den Bunker Nr 13 (11) abgeführt. Das Gespräch des P. Kolbe mit dem Lagerführer Fritsche wurde in deutscher Sprache geführt. Viele von den Anwesenden kannten die deutsche Sprache. Laut übereinstimmenden Aussagen von Zeugen, die das Lager überlebt haben, war der Inhalt des Gespräches der, daß P. Kolbe freiwillig an Stelle des Häftlings Gajowniczek in den Todesbunker gehen will, weil dieser Frau und Kinder hat. Auf Fritschens Frage nach seinen Beruf antwortete P. Kolbe, $\mathrm{da} \beta$ er katholischer Priester ist. Andere Einzelheiten des Gespräches können nicht mit voller Gewissheit festgestellt werden, da sie nur in einigen Berichten vorkommen, z.B., das zweite Motiv des Tausches, daß P. Kolbe gesagt haben sollte, er sei alt und nicht so dringend notwendig im Leben.

Uber das Verhalten P. Maximilians Kolbe im Todesblock berichtet Bruno Borgowiec, der für die unterirdischen Bunker des Blokkes 13 (11) bestellte Funktionär, Folgendes: (27 XII 1945): „Bei den Inspektionen (der im Block 13 diensthabenden SS-Männer) war ich immer anwesend, denn ich mußte die Nummern der Verstorbenen notieren, eventuelle Gespräche oder Bitten der Verurteilten aus dem Polnischen ins Deutsche übersetzen. Aus der Zelle, in welcher diese Armen sich befanden, hörte man jeden Tag lautes Beten, Rosenkranzgebet, Gesang, dem sich auch die Häftlinge aus den Nachbarzellen anschlossen ... Inbrünstige Gebete und Lieder der Unglücklichen zur Mutter Gottes ertönten in allen Korridoren des Bunkers. Ich hatte den Eindruck, ich wäre in einer Kirche. P. Kolbe sagte an, die Häftlinge wiederholten im Chor. Nicht selten waren sie so vertieft im Gebet, daß sie sogar nicht hörten, dass Inspektion abhaltende SS-Männer in den Bunker heruntergekommen waren, und erst auf ihr lautes Schreien verstummten die Stimmen. Wenn die Zelle geöffnet wurde, flehten die Unglücklichen weinend um ein Stück Brot und Wasser. P. M. Kolbe verhielt sich tapfer, bat nicht und jammerte nicht, sprach anderen Mut zu ... Bei jeder Inspektion sah man P. Kolbe, wie er, während alle anderen auf dem Fussboden lagen, in der Mitte stehend oder knieend mit klarem Blick die Ankommenden ansah. Die SS-Männer, die seine Selbstaufopferung kannten, ... sprachen zueinander: 'Der Pfarrer dort ist doch ein ganz anständiger Mensch. So 
einen haben wir hier noch nicht gehabt.' So verstrichen zwei Wochen. In dieser Zeit starb einer nach dem andern bis ... nur noch vier übrigblieben. Der Lagerleitung dauerte das zu lange ... so liessen sie den Vorsteher der Krankenstube kommen, namens Bock ... einen Kriminalverbrecher, der einem jeden nacheinander eine Injektion Karbolsäure in die Ader der linken Hand gab. P. M. Kolbe reichte, mit einem Gebet auf den Lippen, dem Henker seinen Arm. Ich konnte das nicht mitanschauen ... und ging heraus. Sofort, nachdem die SS-Männer mit dem Henker fortgegangen waren, kehrte ich in die Zelle zurück und fand P. M. Kolbe sitzend an die Hinterwand angelehnt, mit offenen Augen und seitwärts gesenktem Kopf" 11 .

Wollen wir vielleicht zunächst die Frage beantworten, warum der heroische Tod des Paters Kolbe während des Beatifikationsverfahrens nicht als ein Märtyrertod anerkannt wurde. Die Ursachen dieser Sachlage waren wohl verschieden. Die wesentlichsten, weit doktrinärer Art, hat P. A. Ricciardi, der Beatifikationspostulator, folgendermaßen formuliert: „Es war sehr schwierig bei den heute geltenden Normen des kanonischen Rechtes das Martyrium für den Glauben $\mathrm{zu}$ beweisen. Die Tatsache des Hasses der Nazis gegen die katholische Religion sind allgemein bekannt. Bestimmt bezeigten ihn auch die, die P. Maximilian zum Tode verurteilten. Aber wenn man als Grund des Hasses diverse Motive annimmt, (religiöse, nationale, u.a.), so ist schwer $\mathrm{zu}$ beweisen, daß im Falle des P. Kolbe der Hass gegen den Glauben die erste und grundsätzliche Ursache des Todesurteiles gewesen ist. Deswegen ist P. Kolbe als Bekenner beatifiziert" 12 .

Es scheint, daß in dieser Ausführung von P. Ricciardi die formalen Elemente des Martyriums zu stark unterstrichen werden, indem er hervorhebt, da $\beta$ der Verfolger in Konzentrationslagern nicht nur aus Hass zum christlichen Glauben und christlicher Sittenlehre mordete und das P. Kolbe nicht aus Treue zu diesen Idealen den Tod hinnehmen mußte. Diese Beweisführung, prinzipiell richtig, scheint jedoch nicht genügend biblisch und christozentrisch dieses schwierige und komplizierte Problem $\mathrm{zu}$ erfassen und deswegen ist sie nicht überzeugend.

Eine gewisse Unkonsequenz in der Auffassung des ganzen Probiems läßt sich bei dem Papst Paul VI. selbst beobachten. Im Widerspruch zu der Seligsprechungsgformel, die über das Märtyrertum vom P. Kolbe schweigt, nannte der Papst den Pater Maximilian einen ,neuen Märtyrer aus Polen" (novello martire della Polonia) in der Homilie, die er während der Beatifikation hielt. Am nächsten Tag (18 X 1971) empfing

${ }_{11}$ I. R. Bar, Smierć O. Maksymiliana Kolbe $w$ świetle prawa kanonicznego, „Prawo Kanoniczne”, 11 (1968), $\mathrm{Nr} 3-4,120-121$. Vgl. ibidem, SS. 111-112, 117. 12 ,Słowo Powszechne", $16-17$ X 1971, S. 6. 
der Heilige Vater polnische Pilger, die nach Rom gekommen waren. In der Anrede an sie sprach er wiederum von dem "Martyrium der Liebe" (martirio di carità) des P. Kolbe ${ }^{13}$.

Aus der Zeit zwischen der Selig- und Heiligsprechung ist auf eine Tatsache hinzuweisen, die, wie es mir scheint, ihren eigenen Beitrag zu der Verleihung des Märtyrertitels dem Pater Kolbe geleistet hat. Das deutsche Messbuch, das vom Heiligen Stuhl akzeptiert wurde, erwähnt unter dem Datum des 14. August, den Seligen Maximilian Kolbe, „Ordenspriester und Märtyrer". Ich glaube, daß es im deutschen Brevier ähnlich ist, doch habe ich es nicht in der Hand gehabt. Wie es dazu gekommen ist, weiß ich einfach nicht. Jedenfalls wurde der Pater Kolbe in dem polnischen liturgischen Kalender nur als Glaubensbekenner bezeichnet. Als vor seiner Heiligsprechung das Problem des Märtyrertums wieder aktuell wurde, war es für diejenigen Menschen in Polen, die in der negativen Beatifikationsentscheidung der römischen Kongregation pro causis sanctorum in dieser Hinsicht den Einfluß seitens der Deutschen vermuteten, ganz überraschend, den Märtyrer Kolbe in dem deutschen Kalender zu entdecken ${ }^{14}$.

Von den vielen an den Apostolischen Stuhl gerichteten Bittgesuchen, in denen gefordert wurde, den P. Maximilian Kolbe während der Heiligsprechung als Märtyrer zu proklamieren, erlaube ich mir lediglich die Punkte 1 und 4 aus dem Schreiben der deutschen und polnischen Bischöfe anzuführen. Sie scheinen nämlich die Ursachen seines Märtyrertums sowohl aus der Sicht der Verfolger (causa martyrii in persecutore), als auch aus der Sicht des Opfers selbst (causa martyrii in martyre) gut zu erfassen. Wir lesen dort folgendes: „1) Die gesamte Ideologie des Nationalsozialismus stand im krassen Widerspruch zu der christlichen Ethik. Er kam vor allem an den Stätten des Massenmordes von vielen Millionen Menschen zum Vorschein, wo mit Vorbedacht alle Menschenund Gottesrechte gebrochen wurden. In so einer Stätte hat der selige Maximilian, der treue Diener der Unbefleckten seinen Tod gefunden;... 4) Die Annahme der freiwilligen Aufopferung zum Tode im Hungersbunker anstelle des Mithäftlings Franciszek Gajowniczek erfolgte erst dann, als die Frage des Lagerführers K. Fritsche mit den Worten: 'Ich bin katholischer Priester' beantwortet wurde. Die Aufopferung des eigenen Lebens für den anderen Menschen war das Zeichen und die Betonung der großen Würde der menschlichen Person und ein Zeugnis der Liebe, deren Beispiel Christus gegeben hat" ${ }^{15}$. Im Vergleich mit der tra-

13 „L'Osservatore Romano", 18-19 Ottobre 1971, S. 1, 2.

14 Der große Wochentagsschot, Teil 2, Herder 1976, S. 1481.

15 L'Osservatore Romano, Wydanie Polskie, $\mathrm{Nr} 9(33)-$ Wrzesień 1982, S. 5. 
ditionellen Auffassung des Martyriums in den Lehrbüchern der Theologie weist die Perspektive des Schreibens der deutschen und polnischen Bischöfe auf das Bedürfnis seiner mehr biblischen und anthropologischen Betrachtung hin, indem aber zugleich die theologischen Hauptelemente seines Wesens beibehalten werden.

Die Frage, was in dem Hungerbunker geschah, wo P. Maximilian Kolbe starb, wird vom Papst Johannes Paul II. selbst gestellt und beantwortet, und zwar in seiner Kanonisationshomilie, die er am $10 \mathrm{X}$ 1982 auf dem St. Petrus-Platz gehalten hat. Es war, seiner Meinung nach, eine besonders getreue Erfüllung der Worte Christi. „Niemand hat eine größere Liebe als (die), wenn jemand sein Leben für seine Freunde aufopfert" (J. 15, 13). Indem P. Maximilian sein Leben für seinen Bruder aufopferte, setzte er sich im Todeslager für das Recht des unschuldigen Menschen zum Leben ein und bestätigte das ausschließliche Recht des Schöpfers auf dieses Leben. Sein Tod gab ihm eine besondere Ähnlichkeit mit Christus, dem Beispiel aller Märtyrer. Durch den Tod Christi auf dem Kreuz wurde die Welt erlöst, weil sein Tod den Wert der höchsten Liebe besitzt. Durch den Tod des Pater Kolbe wurde in unserem auf vielfache Art gefährdeten Jahrhundert ein klares Zeichen dieser Liebe erneuert. Es steckt in diesem menschlich furchtbaren Tod die höchste Größe der menschlichen Wahl- und Tatfreiheit und das Zeugnis in Christus der Menschenwürde, der Heiligkeit seines Lebens, der erlösenden Kraft des Todes, in der die Macht der Liebe erscheint. Daher ist der Tod des P. Kolbe ein Zeichen des Sieges über das ganze Mißachtungs- und Haßsystem dem Menschen gegenüber und ein Zeichen dessen, was im Menschen göttlich ist. Es war ein Sieg diesem ähnlich, den Jesus Christus auf dem Kalvarienberg davongetragen hat.

Nachdem das alles gesagt worden ist, schlußfolgerte der Papst in den letzten Worten seiner Homilie: „Daher habe ich mit meiner vollen apostolischen Gewalt entschieden, daß Maximilian Kolbe, der aufgrund der Seligsprechung als Glaubensbekenner verehrt wurde, von jetzt an auch als Märtyrer verehrt werden soll" ${ }^{16}$.

Karl Rahner schreibt in seiner Abhandlung ,Zur Theologie des Todes”: „Das Martyrium hat es mit dem Tod zu tun”, und „Das Martyrium erscheint als die vollendete, offenbare Erscheinung des christlichen Todes überhaupt". Seiner Meinung nach bedeutet das, daß Gott im Märtyrertode eine grundsätzliche Identität bewirkt und zwar „zwischen dem Sein vor Gott und dem Sein vor der Welt, zwischen der metahistorischen Wahrheit und dem historischen Anschein, zwischen der greifbaren Tat und der inneren Haltung”. Der Märtyrertod ist also ein Tod, „in dem erscheint,

16 „L'Osservatore Romano”, 11-12 Ottobre 1982, S. 1-2. 
was in ihm geschieht" ${ }^{17}$. Eine solche Enthüllung des christlichen Wesens des Todes war, wie jetzt die katholische Kirche glaubt, das Martyrium des heiligen Maximilian Kolbe.

Diesen Betrachtungen mögen noch einige Bemerkungen allgemeinerer Art vom kirchlichen Sinn des Martyriums hinzugefügt werden. Vielfach sind die Beziehungen zwischen einem Märtyrer und der Kirche. Ein Märtyrer bekennt seine Zugehörigkeit zur Gemeinschaft der Gläubigen, er betet und leidet für die Kirche, er ermuntert seine Glaubensgenossen mit Worten, und besonders durch sein Beispiel zur Ausdauer in der Gefahr. Die Kirche bereitet ihrerseits die Gläubigen zur Treue vor, indem sie das Wort Gottes verkündet und Sakramente spendet. So erringen die Märtyrer die nötige Kraft zum Kampf. Im Falle der Not sichert die Kirche dem Märtyrer nach Möglichkeit Schutz bis zur letzten Prüfung, sie begräbt mit Andacht seine sterblichen Überreste, sie schreibt seinen Namen in ihre Kalender ein und erweist ihm religiöse Verehrung ${ }^{18}$.

E. Peterson spricht die Meinung aus, daß das Martyrium organisch mit dem Wesen der Kirche verbunden ist, d.h. daß es mit unbedingter Notwendigkeit einen Bestandteil ihres Begriffes bildet. Was will das bedeuten? Die Verfolgungen sind nämlich in der Kirche nicht etwas Zufälliges und Gelegentliches, sie sind nicht durch Missverständnis oder Irrtum verursacht, d.h. man kann sie nicht einfach nur menschlicher Bosheit oder Arglist zurechnen. Christus hat selbst seiner Kirche Hass und Leiden vorhergesagt. Märtyrer werden sozusagen durch eine Notwendigkeit des Schicksals geschaffen. Die Worte Jesu: „Mußte denn nicht Christus durch solche Leiden in seine Herrlichkeit eingehen?" (Luk 24, 26) beherrschen die ganze Geschichte der Kirche. Sie wird Märtyrer haben, solange das Evangelium der Welt verkündet werden wird, d.i. bis ans Ende der Welt. Natürlich gab es in der Kirche Epochen mit weniger oder mehr Märtyrern, aber eine Behauptung, daß es in der Kirchengeschichte Zeiten ohne Märtyrer gegeben hat, wäre einer Vereinung der Krichenexistenz überhaupt gleichzustellen. Das Zeugnis des Wortes ist somit mit dem Zeugnis des Leidens nicht durch einen gewöhnlichen Zufall verbunden, sondern diese Verbundenheit bildet eine Wirklichkeit, die Christus vorhergesagt hat. Und wer sind die Verfolger? Welches Los ist ihnen be-

${ }_{17} \mathrm{~K}$. R a hner, Zur Theologie des Todes. Mit einem Exkurs über das Martyrium, (Quaestiones disputatae, 2), Herder ${ }^{3}$ 1961, SS. 74, 75, 87, 90.

18 M. Pellegrino, Le sens ecclésial du martyre, ,Revue des sciences religieuses", 35 (1961) 152. Vgl. ibidem 156-174. 
stimmt? Es sind nicht Menschen im allgemeinen abstrakten Sinn, sondern konkrete Menschen, die (um die biblische Terminologie zu gebrauchen) als Juden und Heiden auf Erden leben. Sie sind Feinde des Evangeliums geworden, damit Gott in seiner Barmherzigkeit sich der einen und der anderen erbarme ${ }^{19}$.

Aus dieser Perspektive ist auch das Martyrium des heiligen Pater M. Kolbe in den gemeinsamen deutsch-polnischen Beziehungen zu sehen. Sehr bezeichnend waren in dieser Hinsicht die Worte des verstorben Kardinals I. Döpfner, des Vorsitzenden der deutschen Bischofskonferenz. In der Basilika der Zwölf Apostel in Rom nannte er in einer Rede aus Anlass der Beatifikation P. Kolbe zweimal einen „Märtyrer der Versöhnung" "

Es sei mir erlaubt, noch eine erstaunliche Bemerkung E. Petersons anzuführen. Es geht um römische Grabinschriften, die vom Papst Damasus I. zu Ehren der Märtyrer verfasst worden sind. Sie erinnern u. a. daran, daß in den Verfolgungen der Kirche „ein Schwert das Herz der Mutter durchbohrte", der Mutter Christi. Sie leidet mit den Märtyrern, weil der Leib Christi (vgl. Kol 1, 24) selbst in ihnen leidet. Deshalb gebührt Ihr der Titel: „Königin der Märtyrer” ". Meinerseits darf ich hier hinfügen, daß diese Königin der Märtyrer vom hl. Maximilian Maria Kolbe tiefstens verehrt worden war.

19 E. Peterson, Zeuge der Wahrheit, (Der Märtyrer und die Kirche), in Idem, Theologische Traktate, München 1951, S. 175-178.

20 ,'Tygodnik Powszechny”, 24 X 1971, S. 2.

21 Vgl. Zeuge der Wahrheit, (Der Märtyrer und die Kirche), S. 183. 